\title{
A Hybrid and Adaptive Non-Local Means Wavelet based MRI Denoising Method with Bilateral Filter Enhancement
}

\author{
Kenneth Kagoiya \\ School of Engineering \\ University of Nairobi \\ PO BOX 30197 Nairobi 00100, Kenya
}

\author{
Elijah Mwangi \\ School of Engineering \\ University of Nairobi \\ PO BOX 30197 Nairobi 00100, Kenya
}

\begin{abstract}
Magnetic Resonance Imaging is one of the most advanced and effective medical diagnosis methods ,however the raw image data is normally corrupted by random noise from the measurement process this reduces the accuracy and reliability of the results. Denoising methods are often used to increase the Signal-to-Noise Ratio (SNR) and improve image clarity .In this paper an adaptive Non-Local Means filter is developed in which bilateral filter is used to pre-enhance the images and then multi resolution wavelet domain is used to remove coefficients that contain more noise than signal.

In the past different methods have been used to denoise MRI images but many have not taken into consideration the Rician nature of noise distribution therefore they have not been very effective .Adaptation in this case is based on frequency and spatial information obtained from the noisy image. Knowledge of level of noise is used in an optimization procedure to minimize a Rician based likelihood function and by use of square signal intensity bias is also discarded. The method is implemented in Matlab and MRI images with different level of artificial noise are denoised using the algorithm. Measures of performance values are PSNR, 37.12dB, MSE, 15.23, UQI, 0.985, SSIM, 0.894 , EPI,0.69 for a $10 \%$ noisy image. These and also visual inspection show that there is significant improvement from results obtained using stand alone methods such as Gaussian smoothing, Wiener filter, NLM filter ,bilateral filter and wavelet thresholding.
\end{abstract}

\section{General Terms}

Medical image processing, denoising, measures of quality, magnetization vector, adaptive multi resolution, total variation, wavelet coefficient thresholding ,Non-Local Means, frequency localization, proton spin density, discrete inverse Fourier transform, phase unwrapping, signal-dependent bias, local neighbourhood, Rician-based log-likelihood function.

\section{Keywords}

MRI, Rician noise, wavelet, combinational, NLM, bilateral filter, resolution

\section{INTRODUCTION}

The magnetic resonance image is an intensity coded representation of the structure of an organ or tissue. It is one of the most effective and advanced technologies of medical imaging. The main advantage is that it has no radiation related side-effects unlike other imaging methods. However, a lengthy acquisition time is necessary in order to attain high resolution image. This result in patient discomfort and leads to artifacts related to the body motion of the patient. In addition, noise is also present in the image mainly due to dark current and measurement limitations [1][2][3]. There is therefore a trade-off between high resolution and Signal to Noise Ratio (SNR) [1]. Although averaging may be used to achieve relatively high SNR it is obtained at the expense of spatial resolution. A high resolution image is achieved at the cost of lower SNR thereby making denoising a mandatory step in MR image processing.

Many spatial and transform based denoising methods have been applied to MRI .These include filters based on wavelet coefficient thresholding but have not taken into consideration the Rician nature of the noise and intensity bias thereby reducing their effectiveness. Rician noise causes random fluctuations in the image and also introduces bias that reduces image contrast [1].It degrades images in both qualitative and quantitative senses and hinder image analysis, interpretation and feature detection.

Recent methods have employed the wavelet transform domain but with little consideration of the nature of Rician noise that is prevalent in MRI acquisition. In [4], an adaptive multiresolution block wise non-local means filter is developed for 3D MRI images. This involves adaptive soft wavelet coefficient mixing. In [5] a denoising algorithm for medical images based on a combination of the total variation minimization scheme and wavelet scheme is developed. It involves solution of time evolution partial differential equation by time marching the image using gradient flow resulting in most noise being removed. In [6] [7] [8] thresholding is used together with bilateral filter. In [6] a multi-resolution bilateral filter with wavelet transform subbad mixing is developed.

Analysis based on method noise and also using a generic static image model showed improved performance in comparison to stand alone filters such as Wiener, anisotropic, non-local means, total variation, bilateral and wavelet thresholding. In [10] [11] [12] Non-local non-linear means filters are used. In [10] a set of new similarity measure for NLM filtering are described in which correct Rician statistics of MRI noise are used in image denoising and bias removal. Hybrid filters that constitute two or more filters techniques in parallel or cascade or a more complex combination have recently been used to exploit the merits of each method.

In this paper, a Haar wavelet transform domain approach has been used. The Haar wavelet is used because it perform better than other categories of wavelets in preserving fine image details because it has the most compact spatial support. Although other wavelet transforms may exhibit better frequency localization, the tendency to over smooth an image is a disadvantage.

The rest of the paper is organized as follows. In section 2 a brief presentation of the theory of magnetic resonance imaging is given. In section 3 various denoising filters are 
formulated and section 4 describes the proposed approach while section 5 gives the measures of quality used. Section 6 is on the experimental results.

\section{PHYSICAL PRINCIPLES OF MRI}

In magnetic resonance imaging, the samples are complex signals of the form $S\left(k_{x}, k_{y}\right)$ and are obtained in spatial frequency domain referred to as k-space. These are time domain signal from two independent sources. The signal values in $\mathrm{k}$-space can be expressed as:

$S\left(k_{x}, k_{y}\right)=\iint p(x, y) e^{\left\{-i\left(x k_{x}+y k_{y}\right)\right\}} d x d y$

Where $p(x, y)$ is the spatial distribution of the proton spin density. Filling of $\mathrm{k}$-space is done by designing $\mathrm{k}$-space trajectories as:

$t \rightarrow\left(k_{x}(t), k_{y}(t)=\gamma \int_{0}^{t} G_{x}(\tau) d \tau \int_{0}^{\tau} G_{y}(\tau) d \tau\right.$

Where the function $G_{x}(t)$ and $G_{y}(t)$ are programmable time dependent functions of the magnetic field gradients. The constant $\gamma$ is the gyro magnetic ratio of the image atom nucleus (protons).Thus during an imaging examination $\mathrm{k}$ space is traversed by proper activation of the gradient systems. Usually k-space points are separated by $\Delta k_{x}$ and $\Delta k_{y}$ in two orthogonal directions, and the MRI signals can be sampled on a regular 2-D grid. The spatial distribution of proton spin density $\mathrm{p}(x, y)$ in complex valued image space $(x, y$ ) and the MRI signal $\mathrm{S}\left(k_{x}, k_{y}\right)$ in complex value spatial frequency space define a Fourier pair. Thus the image of proton spin densities can be formed by applying a discrete inverse Fourier transform to the data matrix of k-space samples [5]. Generalization to 3-D imaging involves application of the third gradient coil $\left(\mathrm{G}_{\mathrm{z}}(\mathrm{t})\right)$ in the same fashion. For visual inspection or further processing the magnitude image is used and sometimes also the phase image with possible phase unwrapping. A representation of the acquisition process is the Bloch equation $\partial M / \partial t=\gamma M \times B$ with $B=B_{0}+B_{1}+G$ being the total magnetic field, $B_{o}$ denotes the static magnetic field, $B_{l}$ the excitation pulse and $\mathrm{G}$ the gradient fields. The function $M(r, t)$ is the magnetization vector at position $\mathrm{r}$, rotating at (resonance) frequency $\omega=\alpha \beta$. The total magnetic field $\mathrm{B}$ is under control of the pulse sequence program activating the gradient and RF coils. In the receive mode, these coils are also used to pick up the weak MRI signals.

\subsection{Acquisition and nature of MRI}

The patient is placed in powerful magnet field and electromagnetic waves are passed through the body parts of interest in short pulses where each pulse causes a corresponding pulse of radio waves to be emitted by the patient's tissues. The location from which these signals originate and their strength are determined and a two dimensional image of a section of the tissue is produced [1] [2] [3].

Spatial domain representation of the image is produced by applying Inverse Fast Fourier Transform (IFFT) to the k-space data which results in complex image with a real and imaginary part, this also introduces phase error. There is noise that arises due to random fluctuation in the signal acquisition coil, electronics and Brownian motion in patient which also has a real and imaginary part .In this case both are independently Gaussian distributed [1] [2] processes.

\subsection{Rician Noise}

In [1][3][9] and[17] noise in magnetic resonance magnitude images is shown to obey a Rician distribution. Rician noise is signal-dependent and unlike where noise is Gaussian distributed where spatial and other frequency transform denoising is satisfactorily, separating signal from noise is a difficult task. Removing Rician noise is difficult especially in images with low signal-to-noise ratio (SNR) where there are random fluctuations and a signal-dependent bias to the data that reduces image contrast. In magnetic resonance imaging (MRI), a compromise has to be made among the following, the signal-to-noise ratio (SNR), spatial resolution, and acquisition time required by the intended clinical application. Therefore, given physiological or research paradigm constraints, achievable SNR can be limited. Averaging is a technique which has been used to improve signal-to-noise ratio (SNR). Two types of averaging take place in MRI data acquisition. The discrete nature of the data acquisition process causes spatial volume averaging. In some applications it is common to acquire several measurements at the same location and average them to reduce noise [1].

$$
f(x)=\left\{\frac{x}{\sigma^{2}} I_{0}\left(\frac{m_{x}}{\sigma^{2}}\right) \text { exp }\left(\frac{x^{2}+m^{2}}{-2 \sigma^{2}}\right)\right\} x \geq 0 \text { Rician }
$$

distribution has the form:

$$
I_{0}(y)=\frac{1}{2 \pi} \int_{-\pi}^{\pi} e^{y \cos t} d t
$$

where

is the zeroth order Bessel function of the first kind and $m^{2}=m_{i}^{2}+m_{q}^{2}$

$\sigma$ is the standard deviation of the Gaussian noise distribution that underlines the Rician distribution noise, $\mathrm{m}_{\mathrm{i}}$ and $\mathrm{m}_{\mathrm{q}}$ are the mean values of two(real and imaginary) independent Gaussian components.

\section{DENOISING METHODS}

When established denoising methods are used each on its own they have many shortcomings. In the methods developed in this paper they are used in combination. The mathematical representation of each method is developed in this section.

\subsection{Wavelet Filters}

The multi resolution wavelet transform subdivides the image content into approximation sub-band and a set of detail subbands at different orientations and resolution scales.

The approximation sub-bands consist of scaling coefficients and detail sub bands are composed of the wavelet coefficients. The DWT transform pair is:

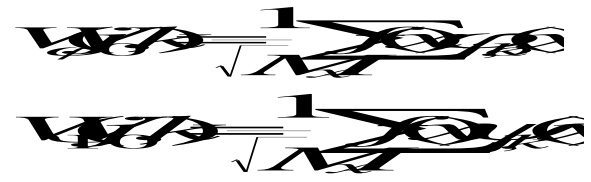

For $j \geq j_{o}$ anc 


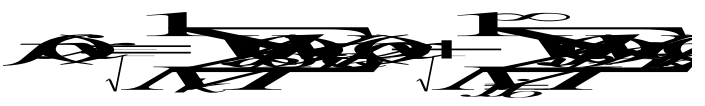

(7)

The one dimensional transforms are extended to twodimensional scaling function, $\varphi(x, y)$, and three twodimensional wavelets functions denoted,

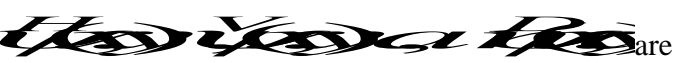

required. Each is the product of a one dimensional scaling function $\varphi$ and corresponding wavelet $\boldsymbol{\psi}$. Excluding products that produce one dimensional results, like $\varphi(x) \psi(x)$, the four remaining products produce the separable scaling function.

Q50)

And separable, "directionally sensitive" wavelets

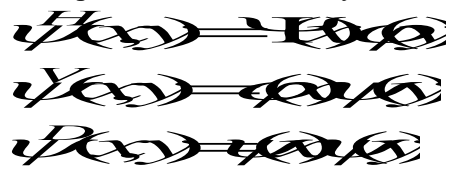

After computation of the two dimensional wavelet transform image denoising in carried out by thresholding or filtering wavelet coefficients.

In magnetic resonance imaging the Rician wavelet based filters give the modified wavelet coefficient [1]

$$
\tilde{d}_{I}=\alpha_{I} d_{I}
$$

With $0 \leq \alpha_{I} \leq 1$ for pixel intensity magnitudes

$$
\alpha_{I}=\frac{d I^{2}-w \sigma^{2} I}{d^{2} I}
$$

Where $\sigma_{I}^{2}$ variance of noisy coefficient and $\mathrm{w}$ is a weighting factor .The modified standard deviation for square image is given by

$$
\tilde{\sigma}_{I}^{2}=4 \sigma_{I}^{2}\left[\sum \varphi_{I}^{2}[m] x^{2}[m]-\sigma_{I}^{2}\right]
$$

Where $\mathrm{m}$ is intensity magnitude

Working with the squared magnitude image is preferred because the wavelet coefficients of the squared magnitude image are unbiased estimators of the wavelet coefficients of the squared signal and the scaling coefficients differ from noise free scaling coefficient by a constant offset not dependent on the signal so it can be easily subtracted.

\subsection{Bilateral filter}

The bilateral filter takes a weighted sum of pixels in a local neighbourhood; the weights depend on spatial distance and intensity distance.

$I(x)=\frac{1}{C} \sum_{y \in N(x)} \ell \frac{-\|y-x\|^{2}}{2 \sigma^{2} d} \quad \frac{-|i(y)-i(x)|^{2}}{2 \sigma^{2} r}$

Where $\sigma_{d}$ and $\sigma_{r}$ are parameters controlling the fall of the weights in special and intensity domains.

$N(x)$ is the spatial neighborhood of $I(x)$ and $C$ is the normalizing constant

$C=\sum y \in N(x) \ell \frac{-\|y-x\|^{2}}{2 \sigma^{2} d} \quad \ell \frac{-|i(y)-i(x)|^{2}}{2 \sigma^{2} r}$

\subsection{Multi-resolution non-local means filtering for MR Image Denoising}

In the formulation of the non-local means filter [4], the restored intensity is a weighted average of the pixel intensities $\mathrm{u}\left(\mathrm{x}_{\mathrm{j}}\right)$ in the search area

$\breve{u}\left(x_{i}\right)=\sum_{x_{j} \in V_{i}} w\left(x_{i}, x_{j}\right) u\left(x_{j}\right)$

With the weighting factor

$w\left(x_{i}, x_{j}\right)=e^{-\frac{\left\|u\left(N_{i}\right)-u\left(N_{j}\right)\right\|}{h^{2}}}$

And for the entire patch the denoised values are:

$\hat{u}\left(N_{i}\right)=\sum_{N_{j} \in V_{i}} w\left(x_{i}, x_{j}\right) u\left(N_{j}\right)$

In magnitude MR images, the distribution of the noise is transformed into a Rician distribution [4],

$p(m)=\frac{m}{\sigma^{2}} \exp \left(-\frac{m^{2}+A^{2}}{2 \sigma^{2}}\right) I_{0}\left(\frac{A m}{\sigma^{2}}\right)$

where $\sigma^{2}$ is the standard deviation of Gaussian noise in the complex domain, $A$ is the amplitude of the noiseless signal, $m$ is the value in the magnitude image and $I_{0}$ is the zeroth order modified Bessel function. The second-order moment of a Rician distribution is given by

$\mathbb{E}\left(m^{2}\right)=A^{2}+2 \sigma^{2}$

In [4], authors proposed a Non-local Maximum Likelihood (NLML) method considering the $\mathrm{N}$ most similar pixels to the current pixel. An optimization procedure is then used to maximize the Rician-based log-likelihood function.

$$
\begin{aligned}
& \log L=\sum_{i=1}^{N} \log \left(\frac{\mathrm{m}_{\mathrm{i}}}{\sigma^{2}}\right)-\sum_{i=1}^{N} \frac{m_{1}^{2}+A^{2}}{2 \sigma^{2}}+ \\
& \sum_{i=1}^{N} \log I_{0}\left(\frac{A m_{i}}{\sigma^{2}}\right) \\
& \hat{A}_{M L E}=\arg \left\{A_{M A X}(\log L)\right\}
\end{aligned}
$$

The conventional approach (CA) is defined as follows

$$
\hat{A}_{C A}=\sqrt{\max \left(\overline{\left.\mathbb{E ( m ^ { 2 }}\right)}-2 \sigma^{2}, 0\right)}
$$

$\left(\widehat{\mathbb{E}\left(m^{2}\right)}\right)$ is estimated as the restored value of square noisy image and $2 \sigma^{2}$ is a fixed signal independent bias which can be removed by subtracting it from each pixel in the magnitude squared image.

$$
\hat{u}\left(N_{i}\right)=\sqrt{\max \left(\left(\sum_{N_{j} \epsilon V_{i}} w\left(x_{i}, x_{j}\right) u\left(N_{j}\right)^{2}\right)-2 \sigma^{2}, 0\right)}(25)
$$

\subsection{Selection of sub-band coefficient and adaptive thresholding}

The sub bands coefficients are described by:

$\hat{c}_{k}=\hat{c}_{k, u}$

$\hat{d}_{k}=\hat{d}_{k, u}$ for LLH, LHL and HLL

$\hat{d}_{k}=\hat{d}_{k, o}$ for HHL, HLH, LHH and HHH

where

$\hat{c}_{k}$ and $\hat{c}_{k, u}$ are the scaling coefficients (i.e. coefficients in LLL low band) of image decompositions, and $\hat{d}_{k}, \hat{d}_{k, u} \hat{d}_{k, o}$ are the wavelet coefficients in detailed sub bands of images $I$ (noisy image), $I_{u}$ (noise removed image) and $I_{o}$ (feature preserved image) decompositions:

The adaptive threshold used $\widehat{T}_{b}(\sigma)$ is computed as for a wavelet coefficients subband $b$ : 


$$
\begin{aligned}
& \hat{T}_{b}(\sigma)=\frac{\sigma^{2}}{\hat{\sigma} X} \\
& \text { with } \\
& \hat{\sigma}_{x}=\sqrt{\max \left(\hat{\sigma}_{b}^{2}-\sigma^{2}, 0\right)}
\end{aligned}
$$

In this approach soft mixing is used instead of hard mixing such that the final coefficient is a weighted average of the coefficients from the two denoised images. This means that: $\hat{c}_{k}=\hat{c}_{k, u}$

and

$\hat{d}_{k}=\varnothing\left(d_{k}, \widehat{T}_{b}(\sigma)\right) \hat{d}_{k, u}+\left(1-\emptyset\left(d_{k}, \widehat{T}_{b}(\sigma)\right)\right) \hat{d}_{k, o}$

with

$\varnothing\left(d_{k}, \widehat{T}_{b}(\sigma)\right)=\frac{1}{1+e^{\left(-\lambda\left(\backslash d_{k} \mid-\widehat{T}_{b}(\sigma)\right)\right)}}$

And the magnitude image is obtained as:

$$
I_{n}\left(x_{j}\right)=\sqrt{I_{r}\left(x_{j}\right)^{2}+I_{i}\left(x_{j}\right)^{2}}
$$

\section{PROPOSED APPROACH}

The algorithm for the proposed method is shown in Figure 1. A bilateral filter is used to diffuse image discontinuities with one image being denoised and the other with all features preserved by edge enhancement. The bilateral filter uses two sets of parameters for the two effects. Wavelet decomposition into various sub bands at different levels is applied to each of the two images. Non-local means filtering is carried out at various sub-bands with appropriate coefficients being computed from original and noisy image statistics. Finally a bilateral filter is used for further enhancement. The input to the filter is either magnitude or square noisy images obtained by adding Rician noise at different levels to a relatively clean image. While working with the models, the parameters $w, \mathrm{~s}_{d}$ and $\mathrm{s}_{r}$ of bilateral filters are varied over a wide range of values as there is no explicit rules that can guide the tuning of these parameters.

\section{MEASURES OF QUALITY}

To assess the effectives of the denoising techniques various measures of quality are used Mean Square Error (MSE) and Peak Signal to Noise Ratio (PSNR) are the first choice but they do not give much information about visual quality. To evaluate image visual quality, additional measures are used. These measures are defined as follows.

\subsection{Signal to Noise Ratio}

This is a measure of signal purity defined as :

$S N R=$
$10 \log _{10}\left(\frac{\sum_{x_{i} \epsilon \Omega} I\left(x_{i}\right)^{2}}{\sum_{x_{i} \epsilon \Omega}\left(I\left(x_{i}\right)-\hat{I}\left(x_{i}\right)\right)^{2}}\right)$

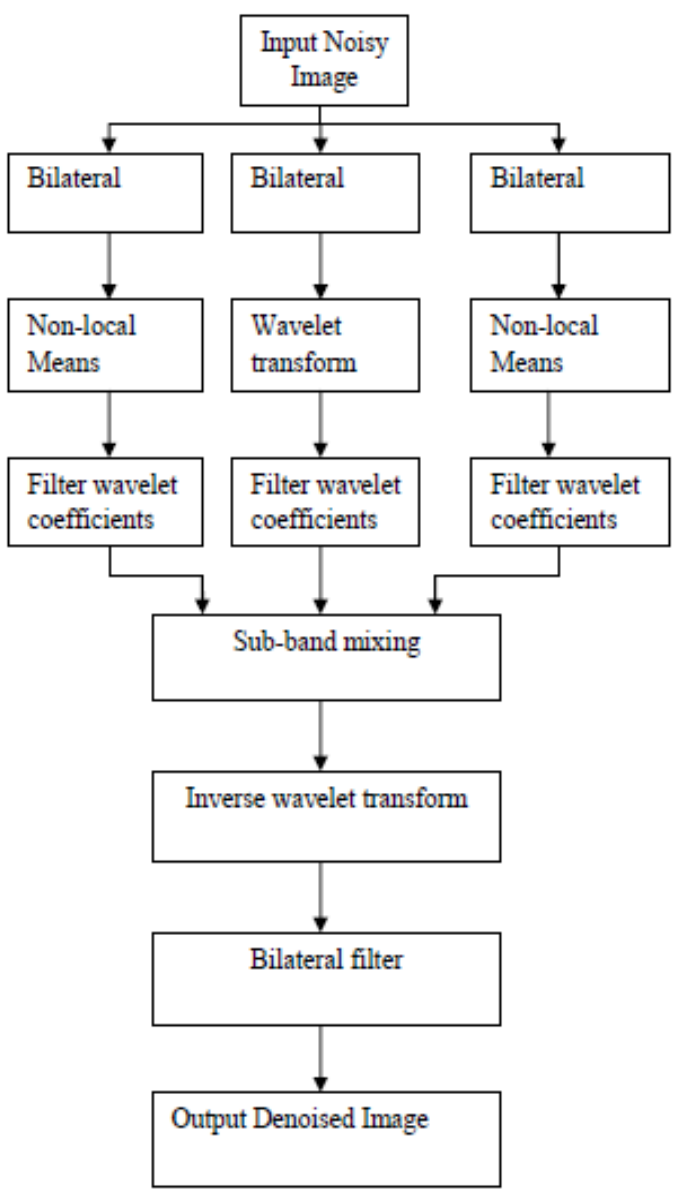

Fig 1 A Flowchart of the Proposed Method

\subsection{Mean square error}

The MSE on gradient is given by

$$
\operatorname{MSE}=\frac{1}{|\Omega|} \sum_{x_{i} \in \Omega}\left(\Delta I\left(x_{i}\right)-\Delta \hat{I}\left(x_{i}\right)\right)^{2}
$$

This metric is based on the "ground truth" image $I$ and the denoised image $\hat{I}$.

\subsection{Correlation Coefficient}

Another useful measure used is the correlation coefficient defined as follows:

$$
\operatorname{CoC}=\frac{\sum_{x_{i} \in \Omega}\left(I\left(x_{i}\right)-\bar{I}\right)\left(\hat{I}\left(x_{i}\right)-\bar{I}\right)}{\sqrt{\sum_{x_{i} \in \Omega}\left(I\left(x_{i}\right)-\bar{I}\right)^{2}} \sum_{x_{i} \in \Omega}\left(\hat{I}\left(x_{i}\right)-\bar{I}\right)^{2}}
$$

\subsection{Edge Preservation Index}

This is a numerical measure of edge profile consistency which is formulated as follows:

$$
E P I=\frac{\sum_{x_{i} \in \Omega}\left(\nabla I\left(x_{i}\right)-\overline{\nabla I}\right)\left(\nabla \hat{I}\left(x_{i}\right)-\overline{\nabla \hat{I}}\right)}{\sqrt{\sum_{x_{i} \in \Omega}\left(\nabla I\left(x_{i}\right)-\nabla \bar{I}\right)^{2}} \sum_{x_{i} \in \Omega}\left(\nabla \hat{I}\left(x_{i}\right)-\nabla \overline{\hat{I}}\right)^{2}}
$$

\subsection{Universal Quality Index}

The Universal Quality Index (UQI), is defined as a product of three factors: loss of correlation, luminance distortion, and contrast distortion as:

$$
U Q I=\frac{\sigma_{I \hat{I}}}{\sigma_{I} \sigma_{\hat{I}}} \cdot \frac{2 \hat{I} \hat{I}}{(\bar{I})^{2}+(\overline{\hat{I}})^{2}} \cdot \frac{2 \sigma_{I \sigma_{\hat{I}}}}{\sigma_{1}^{2}+\sigma_{\hat{I}}^{2}}
$$




\subsection{The Mean Structural Similarity Index Measure (MSSIM)}

This is a measure of closeness in structure of two images. It is defined as:

$$
M S S I M=\frac{\left(2 \hat{I} \hat{I}+C_{1}\right)\left(2 \sigma_{I I}+C_{2}\right)}{\left((\bar{I})^{2}+(\hat{I})^{2}+C_{1}\right)\left(\sigma_{I}^{2}+\sigma_{I}^{2}+C_{2}\right)}
$$

where $\bar{I}$ and $\hat{\bar{I}}$ are the means of the noise-free and the denoised image and $C_{1}, C_{2}$ are correlations respectively.

These measures of quality require data on the noisy and noiseless image as well as images gradient for computation of edge preservation index and mean square error on gradient. Appropriate scaling is required so that truncation of high square values of intensity is avoided.

\section{EXPERIMENTAL RESULTS}

The image data used in the experimental investigations have been sourced from three main public repositories. BrainWeb [26], Luisier [27] and Clunie [28]. These are the main libraries for MRI data have been used by many investigators due to their ease of availability. These images were acquired using a Siemens Vision scanner echo-planar imaging system with a $1.5 \mathrm{~T}$ magnetic field. Other parameters of the scanner being field of view $(\mathrm{FOV})=240 \mathrm{~mm} \times 240 \mathrm{~mm}$, relaxation time $(\mathrm{TR})$ $=2000 \mathrm{~ms}$, echo time $(\mathrm{TE})=$ flip angle $(\mathrm{FA})=90^{\circ}$, for $256 \times 256$ image sizes.To compare the effectiveness of the adaptive combination non-local means filter to the stand alone state of the art denoising methods, Rician noise at various levels from $2 \%$ to $20 \%$ is added to noise free magnetic resonance image.The levels are chosen because acquired images noise would be in this range.The images used include Torso1, Torso2 and Hip which are structural 2-D images.

\subsection{Effect of noise addition}

The visual effect of noise addition was tested with three structural 2-dimensional MR images taken from the torso and the hip. We have referred to these as torsol, torso2, and hip. These three images are displayed in Fig 2 priori to Rician noise addition. These images have features that are very clear and the edges are also relatively distinct. They also have a wide intensity range from completely dark to complete white, that is in the pixel intensity range ( 0 to 256) for an 8 bit system. They also exhibit high and low frequency regions. For the purpose of algorithm testing, the images have been resized from various sizes to $256 \times 256$

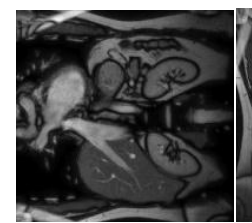

(a)

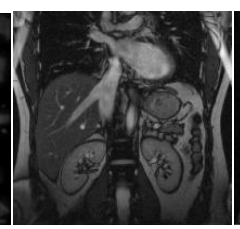

(b) (c)

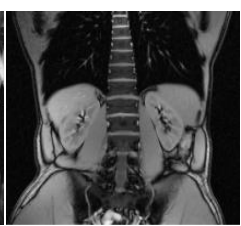

Fig 2 Noise-free MRI images (a) Torso1, (b) Torso2, (c) Hip

The addition of Rician noise on the visual quality of the images is illustrated in Fig 3(a), (b), (c) and (d) in regard to the image torsol. The level of noise in the image was varied from $2 \%$ to $5 \%$ to $10 \%$ up to $20 \%$. It can be noted that for a low level of noise degradation, the image edges are still very clear but contrast can been seen to have been reduced slightly. This could affect the interpretation of small features in the image during diagnosis; the same applies to 5\% though contrast is worse. At $10 \%$ it is difficult to distinguish the boundary of some tissue and the random noise is clearly visible. At $20 \%$ only the major features are recognizable with minute features buried in the random noise. Similar observations were made with the torso 2 and hip images.

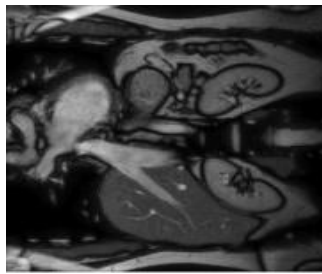

(a) $\mathbf{2 \%}$

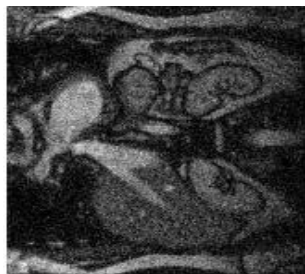

(c)10\%

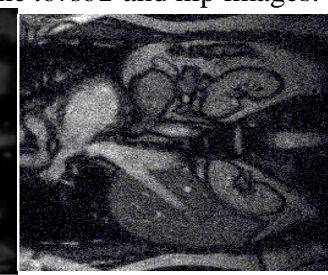

(b) $5 \%$

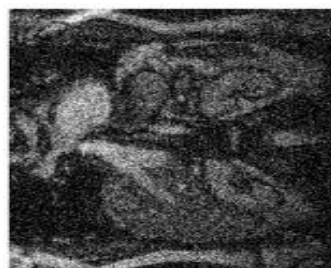

(d) $20 \%$
Fig 3 Noisy images at given levels for torso1

\subsection{Denoising using Thresholding, Median, Bilateral and Non-local methods}

The results of employing the stand alone methods in noise suppression is illustrated in Figure 4.This is without taking advantage of the contribution of each filter in the combination. Using the bilateral alone may lead to over smoothing hence obscuring fine details; using wavelet thresholding on its own also leads to loss of fine details. Figure 4 (a) shows extreme whitening and darkening when thresholding is used. This is because below a given intensity value all signal is assumed to be noise and truncated and the bias in the magnitude image takes intensity values above a certain value to maximum. This upper threshold is the difference between the maximum value and the bias. In Figure 4 (b) it can be observed that the bilateral filter and median filter when used each on its own lead to smoothing of image details. Figure4 (c) shows that the nonlocal filter on its own gives a relatively better image but the contrast and the boundaries clarity needs further improvement.

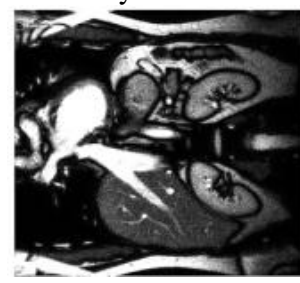

(a) Thresholding

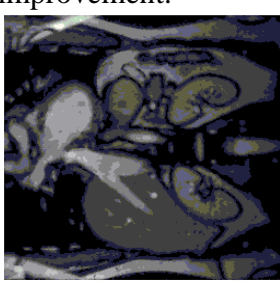

(b) Median
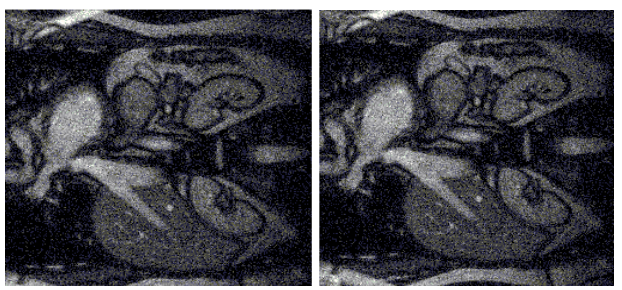

(c )Non-local means (d) Bilateral Fig 4 Denoised images using other methods 


\subsection{Denoising using the proposed method}

The proposed method is shown in figure 1 where a bilateral filer is used to preserve edges before the main method; Nonlocal means with soft wavelet thresholding is applied .Fig 5 shows the result of using the proposed algorithm. At $2 \%$ and $5 \%$ noise addition, the recovery process is very efficient as can be noted from a visual inspection of the images. For example, at $2 \%$ noise addition the recovered image is seen to be almost the same as the noiseless image. The observations are also analyzed by employing the mathematical quality measures described in section 3 and given in table 1, 2, 3, and 4. For example in Table 1 and Table 2 where SNR is very high, MSE relatively low ,UQI almost 1, SSIM 0.984 and EPI 0.89 which is an improvement from 0.70 of the noisy image. It also shows that edge preservation is very sensitive even for low noise. The use of bilateral filter in this combination algorithm has been shown to be the main contributor for edge preservation.

The results obtained when the five measures of quality are used to assess the effectiveness of image torsol denoising using the proposed method for relatively low noise levels are shown in table 1 and table 2 .

Table 1. Quality measures at 2\%

\begin{tabular}{|c|l|l|l|l|l|}
\hline $\begin{array}{c}\text { Noise Filter } \\
\text { Type }\end{array}$ & $\begin{array}{c}\text { PSNR } \\
\text { DB }\end{array}$ & RMSE & UQI & SSIM & EPI \\
\hline Noisy Image & 38.04 & 16.23 & 0.96 & 0.86 & 0.70 \\
\hline Median & 41.23 & 13.55 & 0.97 & 0.89 & 0.75 \\
\hline Wiener & 42.38 & 11.12 & 0.97 & 0.97 & 0.74 \\
\hline Bilateral & 44.02 & 8.654 & 0.99 & 0.98 & 0.85 \\
\hline Thresholding & 38.54 & 22.43 & 0.95 & 0.93 & 0.68 \\
\hline NLM & 43.54 & 8.54 & 0.98 & 0.90 & 0.82 \\
\hline Combinational & $\mathbf{4 4 . 4 3}$ & $\mathbf{6 . 4 5}$ & $\mathbf{0 . 9 9}$ & $\mathbf{0 . 9 8}$ & $\mathbf{0 . 8 9}$ \\
\hline
\end{tabular}

Table2. Quality measures at $5 \%$

\begin{tabular}{|c|c|c|c|c|c|}
\hline $\begin{array}{c}\text { Noise Filter } \\
\text { Type }\end{array}$ & $\begin{array}{c}\text { PSNR } \\
\text { DB }\end{array}$ & $\begin{array}{c}\text { RMS } \\
\text { E }\end{array}$ & UQI & $\begin{array}{c}\text { SSI } \\
\text { M }\end{array}$ & EPI \\
\hline Noisy Image & 35.02 & 23.53 & 0.95 & 0.84 & 0.62 \\
\hline Median & 37.64 & 18.45 & 0.98 & 0.89 & 0.72 \\
\hline Wiener & 38.56 & 12.34 & 0.99 & 0.95 & 0.71 \\
\hline Bilateral & 41.23 & 8.654 & 0.98 & 0.95 & 0.80 \\
\hline Thresholding & 36.54 & 34.12 & 0.87 & 0.84 & 0.71 \\
\hline NLM & 39.54 & 14.23 & 0.99 & 0.95 & 0.82 \\
\hline Combinational & $\mathbf{4 2 . 3 8}$ & $\mathbf{1 2 . 5 5}$ & $\mathbf{0 . 9 5}$ & $\mathbf{0 . 9 5}$ & $\mathbf{0 . 8 4}$ \\
\hline
\end{tabular}

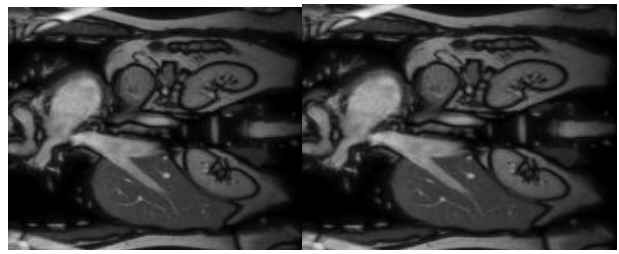

(a) $2 \%$

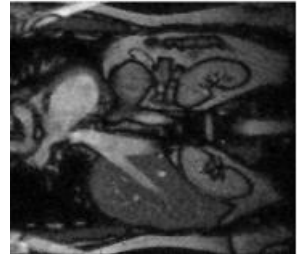

(c) $10 \%$ (b) $5 \%$

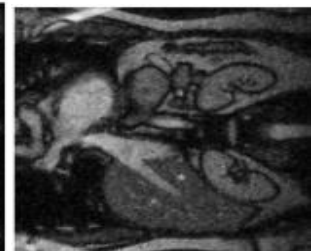

(d) $20 \%$
Fig 5 Denoised images (new algorithm)
The quality measures have also been used to give an assessment of the simulation results for $10 \%$ and $20 \%$ noise levels.

Table 3. Quality measures at $10 \%$

\begin{tabular}{|c|c|c|c|c|c|}
\hline $\begin{array}{c}\text { Noise Filter } \\
\text { Type }\end{array}$ & $\begin{array}{c}\text { PSNR } \\
\text { DB }\end{array}$ & $\begin{array}{c}\text { RMS } \\
\text { E }\end{array}$ & UQI & $\begin{array}{c}\text { SSI } \\
\text { M }\end{array}$ & EPI \\
\hline Noisy Image & 29.52 & 35.45 & 0.80 & 0.74 & 0.48 \\
\hline Median & 31.21 & 25.34 & 0.87 & 0.84 & 0.57 \\
\hline Wiener & 33.25 & 19.95 & 0.97 & 0.87 & 0.51 \\
\hline Bilateral & 34.53 & 16.78 & 0.98 & 0.89 & 0.60 \\
\hline Thresholding & 28.51 & 45.67 & 0.95 & 0.84 & 0.52 \\
\hline NLM & 36.45 & 17.76 & 0.98 & 0.89 & 0.68 \\
\hline Combinational & $\mathbf{3 7 . 1 2}$ & $\mathbf{1 5 . 2 3}$ & $\mathbf{0 . 9 9}$ & $\mathbf{0 . 8 9}$ & $\mathbf{0 . 6 9}$ \\
\hline
\end{tabular}

Table 4. Quality measures at $20 \%$

\begin{tabular}{|c|c|c|c|c|c|}
\hline $\begin{array}{c}\text { Noise Filter } \\
\text { Type }\end{array}$ & $\begin{array}{c}\text { PSNR } \\
\text { DB }\end{array}$ & RMSE & $\begin{array}{c}\text { UQ } \\
\text { I }\end{array}$ & $\begin{array}{c}\text { SSI } \\
\text { M }\end{array}$ & EPI \\
\hline Noisy Image & 23.45 & 46.72 & 0.85 & 0.65 & 0.34 \\
\hline Median & 24.20 & 30.94 & 095 & 0.72 & 0.37 \\
\hline Wiener & 26.24 & 25.43 & 0.96 & 0.73 & 0.44 \\
\hline Bilateral & 27.54 & 22.69 & 0.96 & 0.80 & 0.41 \\
\hline Thresholding & 23.21 & 57.31 & 0.95 & 0.73 & 0.38 \\
\hline NLM & 26.78 & 23.47 & 0.96 & 0.88 & 0.45 \\
\hline Combinational & $\mathbf{2 8 . 6 9}$ & $\mathbf{1 9 . 2 3}$ & $\mathbf{0 . 9 7}$ & $\mathbf{0 . 8 8}$ & $\mathbf{0 . 4 7}$ \\
\hline
\end{tabular}

Tables 1 to tables 4 show that the adaptive non-local means filter outperform other methods like Thresholding and Wiener alone when used with multi-resolution wavelet soft thresholding. The inclusion of bilateral filter contributes to overall effectiveness not only on edge preservation but also on SSIM and MSE. In Table 4, where the added noise is $20 \%$, the quality measures show that the efficiency and effectiveness of all the denoising methods is relatively low. It is therefore necessary that a relatively clear image is reconstructed during the acquisition process.

\subsection{Residue Noise}

Figure 6 shows noise image which is the difference between noiseless (reference) image and noisy image. Result show that noise is signal dependent and even after denoising the slight residual noise has a remote relationship with the image.

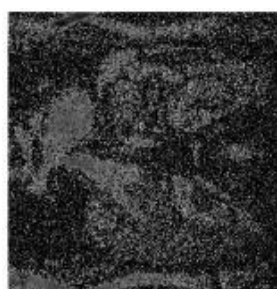

(a) Before denoising

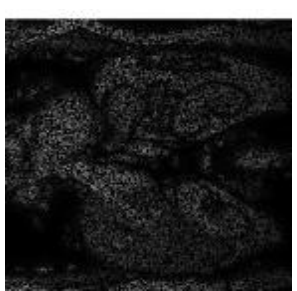

(b) After denoising

Fig 6 Noise image

\section{DISCUSSION AND CONCLUSIONS}

The denoising results obtained for each of the tested images using the combinational adaptive NLM filters and those of the standalone filters have been obtained by computer simulation and given in figure 4 and figure 5 and in table 1, 2, 3, and 4 . From these figures, it can be seen that the proposed combinational algorithm result in higher-contrast denoising and better visual clarity as compared to the stand-alone methods. The proposed method also suppresses image noise to a higher level that the other methods. The PSNR, RMSE, 
UQI, SSIM and EPI quality measures have been used to provide a comparison of the noise reduction levels by the stand alone filters and the proposed method.

It has been shown that using the proposed method there is some significant improvement over the other methods in image quality while exhibiting higher effective resolution and contrast. The results obtained in a high noise regime indicate poor recovery of the original image. This has been evidenced by our simulations as given in fig 5 (c) and in tables 3 and 4 where at least $10 \%$ noise addition was used. The solution lies in the improvement of the measurement and calibration system of MRI equipment. This may be achieved by use of more gradients and use of parallel acquisition methods. Additional improvement could also be obtained by slice time correction and motion correction.

\section{REFERENCES}

[1] R. Nowak, "Wavelet-based Rician noise removal for Magnetic Resonance Imaging," IEEE Transactions on Image Processing, vol. 8,no. 10, pp. 1408-1419, October 1999.

[2] T. Dylan "MRI denoising via phase error estimation" medical imaging journal proc on image processing SPIE Vol5 7472005

[3] Santiago, "Restoration of DWI data using a Rician LMMSE Estimator", IEEE Transactions on on medical imaging, Vol, 27, No, 10, October 2008.

[4] J. V. Manj'om, P. Coup'e, L. Marti-bonmati, M. Robles, and D. L. Collins, "Adaptive non-local means denoising of MR images with spatially varying noise levels," Journal of Magnetic Resonance Imaging, vol. 31, pp. 192-203, 2010

[5] M. Lysaker, A. Lundervold, and X. C. Tai, "Noise removal using fourth-order partial differential equation with applications to medical magnetic resonance images in space and time," IEEE Transactions on Image Processing, vol. 12, no. 12, pp. 1579-1590, December 2003.

[6] Z. A. Mustafa, Y. M. Kadah, "Multiresolution Bilateral Filter for MR Image Denoising ," Biomedical Engineering Department, Cairo University, IEEE 2011.

[7] R .Sudipta, "A new hybrid image denoising method", International Journal of Information Technology and Knowledge Management July-December 2010, volume 2,p 491-497.

[8] V. Loganayaagi, “An improved Denoising Algorithm Using Wavelet Transform for Magnetic Resonance Images", International journal of Communications and Engineering Volume 07-No 7,Issue 01 march2012.

[9] A. Pizurica, A. M. Wink, E. Vansteenkiste, W. Philips, and J. B. T. M. Roerdink, "A review of wavelet denoising in MRI and ultrasound brain imaging," Current Medical Imaging Reviews, vol. 2, no. 2, pp. 247-260, May 2006.

[10] S. Dolui, A. Kuurstra, C. Iv'an. Salgado Patarroyo and Oleg V. Michailovich "A New Similarity Measure for Non-Local Means Filtering of MRI Images," October 28 2011

[11] T. Tasdizen "Principal Neighborhood Dictionaries for Non-local Means Image Denoising," IEEE Transactions on Image Processing, Vol. xx, No. x, January 2009.
[12] P. Coup'e, P. Yger, S. Prima, P. Hellier, C. Kervrann, and C. Barillot, "An optimized block wise nonlocal means denoising filter for 3-D magnetic resonance images," IEEE Transactions on Medical Imaging, vol. 27, no. 4, pp. 425-441, March 2008

[13] C. Lakshmi Devasena "Noise Removal in Magnetic Resonance Images using Hybrid KSL Filtering Technique," International Journal of Computer Applications (0975 - 8887) Volume 27- No.8, August 2011.

[14] Palaniappan "Denoising of dynamic magnetic resonance images by combined application of wavelet filtering and Karhunen-Loeve Transform (KLT)", Journal of Cardiovascular Magnetic resonance 2012 14(sup1) W71.

[15] J. V. Manjon "Multi-component MR image denoising", International Journal of Biomedical imaging volume 2009, article ID756897.

[16] M. Zhang and B. K. Gunturk, "Multiresolution Bilateral Filtering for Image Denoising", IEEE Trans Image Process, vol. 17,pp. 2324-2333,2008.

[17] F. Luisier, T. Blu, P. J. Wolfe. "A Cure for Noisy Magnetic Resonance Images: Chi-square Unbiased Risk Estimation”, Harvard University; 15 June 2011.

[18] A. Pizuria J Adtormann, B .Gossens, W. Phillips "Removal of Correlated Rician Noise in Magnetic Resonance Imaging", $16^{\text {th }}$ European Signal processing Conference (EUSIPCO 2008), Lausanne, Switzerland, August 25- 292008.

[19] A.K .Jain "Fundamentals of digital image processing", Prentice hall Engle wood cliffs, NJ USA 1989

[20] A Pizuria, Philips A W Lemahieu and Acheroy M “ A versatile wavelet domain noise filtration technique for medical imaging”, IEEE Trans Med Imaging 2003, 22(3) pp 232-331

[21] Hossein "Wavelet Domain medical image denoising using Bivariate laplacian mixture model", IEEE Transactions on biomedical engineering vol 56 No 12 December 2009

[22] J. V. Manjón, Pierrick Coupe, Antoni Buades. "MRI Noise Estimation and Denoising Using Non-Local PCA.’" Medical Image Analysis, 22:35-47. 2015.

[23] J. V. Manjón, Simon F. Eskildsen, Pierrick Coupé, Jose E. Romero, D. Louis Collins, Montserrat Robles. "Nonlocal Intracranial Cavity Extraction. IJBI." Article ID 820205. 2014.

[24] J.V. Manjon, Pierrick Coupe, Luis Concha, Antonio Buades, D. Louis Collins, Montserrat Robles. "Diffusion Weighted Image Denoising using overcomplete Local PCA." PLoS ONE 8(9): doi:10.1371/journal.pone.0073021. 2013

[25] P. Coupé, J. V. Manjon, M. Chamberland, M. Descoteaux. "Collaborative patch-based super-resolution for diffusion-weighted images." 'NeuroImage, 83:245261,2013

[26] www.braiweb.bic.mni.mcgill.ca/brainweb June.15, 2014

[27] http://bigwww.epfl.ch/luisier/MRIdenoising/Test Images.zip June.15, 2014

[28] http://www.dclunie.com/ June.15, 2014 